\title{
An experimental demonstration of blind ocean acoustic tomography ${ }^{\text {a) }}$
}

\author{
Sérgio M. Jesus ${ }^{\text {b) }}$ and Cristiano Soares \\ SiPLAB - FCT, Universidade do Algarve, Campus de Gambelas, PT-8005-139 Faro, Portugal \\ Emanuel Coelho \\ NATO Undersea Research Centre, Viale San Bartolomeo 400, I-19138 La Spezia, Italy \\ Paola Picco \\ ENEA, Marine Environment Research Centre, P.O. Box 224, I-19100 La Spezia, Italy
}

(Received 23 June 2005; revised 13 December 2005; accepted 14 December 2005)

\begin{abstract}
Despite the advantages clearly demonstrated by ocean acoustic tomography (OAT) when compared to other ocean monitoring techniques, it suffers from several technical-related drawbacks. One is the requirement for rather expensive equipment to be maintained and operated at several locations in order to obtain sufficient source-receiver propagation paths to cover a given ocean volume. This paper presents the preliminary feasibility tests of a concept that uses ships of opportunity as sound sources for OAT. The approach adopted in this paper views the tomographic problem as a global inversion that includes determining both the emitted signal and the environmental parameters, which is a similar problem to that seen in blind channel identification and was therefore termed blind ocean acoustic tomography (BOAT). BOAT was tested on a data set acquired in October 2000 in a shallow-water area off the west coast of Portugal, including both active and passive (ship noise) data. Successful results show that BOAT is able to estimate detailed water column temperature profiles coherent with independent measurements in intervals where the uncontrolled source signal (ship noise) presents a sufficient bandwidth and signal-to-noise ratio, which clearly define the limitations of the presented method. (c) 2006 Acoustical Society of America.
\end{abstract}

[DOI: $10.1121 / 1.2165070]$

PACS number(s): 43.30.Wi, 43.30.Pc, 43.60.Cg, 43.60.Pt [AIT] Pages: 1420-1431

\section{INTRODUCTION}

Ocean acoustic tomography (OAT) was proposed more than three decades ago but its routine usage is still not a reality today. Despite the advantages clearly demonstrated by OAT when compared to other ocean monitoring techniques, it suffers from several drawbacks, such as the requirement for rather expensive equipment to be maintained and operated simultaneously at several locations in order to obtain sufficient source-receiver propagation paths to cover a given ocean volume. In particular, OAT is known to be very sensitive to the precise knowledge of source-receiver relative locations at all times. Passive acoustic tomography (PAT) is an acoustic tomography variant where the usual cooperative acoustic source is replaced by a noncooperative noise source as for example a ship of opportunity. The basic idea behind PAT is to extend the application of acoustic tomography to areas with heavy or regular ship traffic and where it would be impossible, or too costly, to deploy a controlled acoustic source in a permanent basis. PAT is also an interesting alternative to active tomography in the presence of marine mammals or for covert military application. The broad concept of using alternative illuminating sources for OAT or for geoacoustic inversion is not new. It was first proposed by Buck-

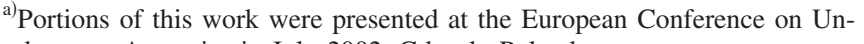
derwater Acoustics in July 2002, Gdansk, Poland.

b) Electronic mail: sjesus@ualg.pt
}

ingham to use acoustic daylight to form images of silent objects in the ocean ${ }^{1,2}$ and then using ambient noise for geoacoustic inversion. ${ }^{3}$ More recently, Harrison ${ }^{4-6}$ used sea surface wind-induced noise; then, Buckingham et al. ${ }^{7}$ used light aircraft air-induced noise, both with the purpose of shallowwater geoacoustic inversion.

PAT differs from classic active acoustic tomography by the fact that in PAT, the source signal is stochastic with unknown characteristics and uncontrolled by the experimenter. There are at least two important implications of the assumptions made under PAT: one is that the emitted signal is possibly fluctuating over time both in strength and bandwidth, the other is that the sound source's position is (in general) unknown and possibly changing over time. The fact that the source position is unknown implies that, apart from the sound-speed profile to be inverted for, the other propagation channel characteristics (e.g., bottom properties, water depth, etc.) are also unknown and have to be estimated together with the source position. An inverse problem where both the input signal and the channel are unknown is termed a blind deconvolution problem, and is common in the fields of wireless communications, geophysics, and in all problems where channel identification is required and where the input signal is not known (see Cadzow $^{8}$ for an overview). The generally adopted methodology is to use higher-order statistics and (in wireless communications) the cyclostationarity properties of the received signal. ${ }^{9,10}$ Such methods have also been used in 
underwater acoustics for signals with some degree of nonstationarity. ${ }^{11,12}$ Assuming that the noise sources of opportunity are relatively stationary inputs to the propagation channel, it is possible to build a model-based cost function where both the source and the channel properties are unknown variables to be estimated. In essence, due to the highly random and incoherent nature of the signal phase along the propagation path, the actual emitted waveform is almost never used for matched-field processing at the receiver. The exception to this is of course coherent underwater communications. It is therefore sufficient to consider a second-order cost function working in a frequency band where the signal is assumed to propagate. Using this analogy between blind deconvolution in passive ocean tomography, this technique was termed blind ocean acoustic tomography (BOAT). The distinction between PAT and BOAT is that the former aims at estimating ocean temperature with alternative passive sources, while the latter produces a full environmental estimate, including water column, bottom properties, and source-receiver geometry as well as a source-emitted power spectrum, without any knowledge or control on the acoustic illuminating source. Therefore, BOAT deals both with the emitting source power spectrum and with the estimation of a full environment model response, often described by a variable but large number of physical parameters.

The hardest problem in BOAT is dealing with a large number of parameters. Difficulties are associated not only with the number of parameters but also with the different nature of the parameters in play, where geometrical, water column, and bottom parameters are simultaneously searched for. An additional difficulty arises from the fact that our degree of knowledge of the parameters to be inverted for may be highly variable. Several approaches have been proposed to deal with such inverse problems either for geoacoustics, ${ }^{13-16}$ or for the water column. ${ }^{17,18}$ In other studies the parameters under search simultaneously included geoacoustic, water column, and geometric parameters both known and unknown. ${ }^{19,20}$ The term generally used for approaches performing multiparameter search including both known and unknown parameters is focalization, and was first proposed by Collins et al. $^{21}$ in the context of range-depth source localization. So, in our case the problem to be addressed under the scope of this paper involves an environmental and geometrical focalization procedure with a random source of unknown spectra. It is well known that conventional matched-field processing (MFP) Bartlett-based cost functions are suboptimal when dealing with highly variable (nonflat frequency spectrum) power sources. ${ }^{22}$ An alternative procedure for estimating also the source-emitted spectra using the estimates as weighting function for the conventional MFP-based cost function was proposed by the authors in Ref. 23 and is used here in the INTIFANTE' 00 data set.

The INTIFANTE'00 sea trial was a joint experiment carried out by Instituto Hidrográfico and the University of Algarve and the collaboration and support of several other institutions [Insituto Superior Técnico (IST), Lisboa, Portugal, Ente per l'Energia ed l'Ambiente (ENEA) and SACLANT Undersea Research Centre, both in La Spezia, Italy] in the

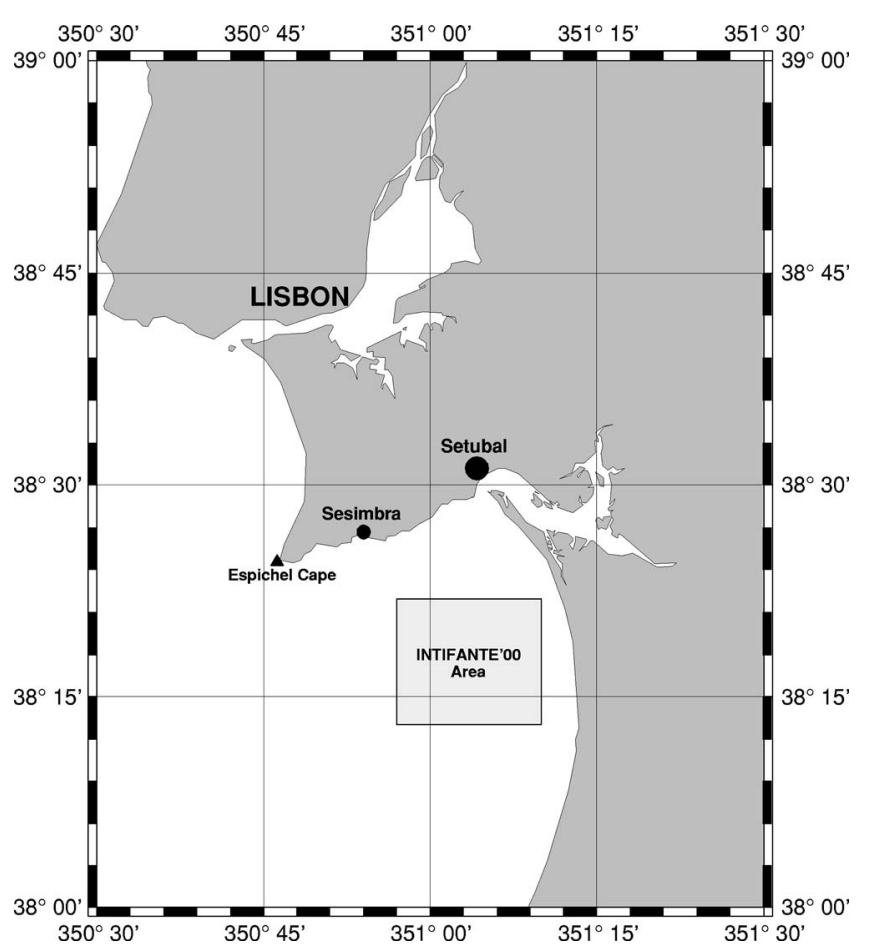

FIG. 1. INTIFANTE'00 sea trial location.

fall of 2000, in a shallow-water area $50 \mathrm{~km}$ south from Lisbon, Portugal. There was a broad range of objectives pursued by this experiment, among which the testing of the ability of inverting ocean properties with both known and unknown, active and passive, stationary and moving acoustic source signals in various environments, both range independent and range dependent. The results shown in this paper cover the following situations: range independent and range dependent with a moving unknown active source, and a fast-moving ship acting as source in a partially range-dependent environment.

This paper is organized as follows: Sec. II gives an overview of the INTIFANTE'00 experiment. Section III presents the environmental model drawn from the direct measurements performed during the sea trial. Methods and algorithms used during the processing of the data are described in Sec. IV. Section V describes the results obtained with a towed active source and a ship, the research ship NRP D. Carlos I, both assumed unknown. Final conclusions and perspectives are drawn in Sec. VI.

\section{THE INTIFANTE'00 SEA TRIAL}

The INTIFANTE'00 (INTIFANTE is a made-up acronym from INTImate and inFANTE, two cofunding projects) sea trial was carried out in the vicinity of Setúbal, situated approximately $50 \mathrm{~km}$ to the south of Lisbon, in Portugal, during the period from 9 to 29 October, 2000 (Fig. 1). A detailed and complete description of the experiment and of the various data sets acquired during the INTIFANTE'00 sea trial can be found in Ref. 24, while here only a brief description will be given. The experiment area was a rectangular box situated within the continental shelf with depths varying from 60 to $130 \mathrm{~m}$. The actual acoustic runs were performed 


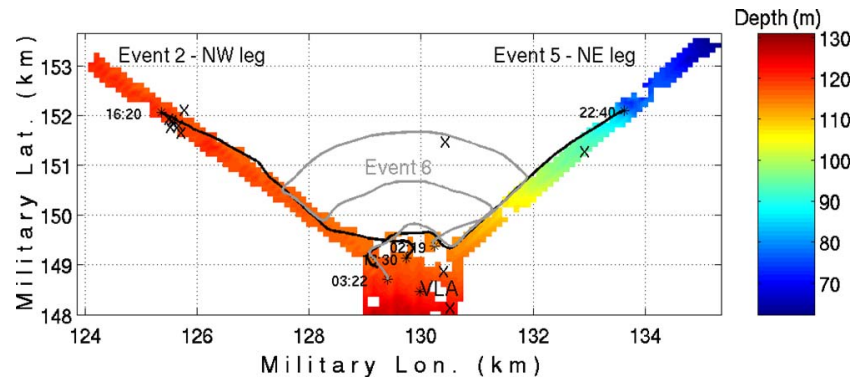

FIG. 2. (Color online) INTIFANTE'00 sea trial: acoustic runs and bathymetry during events 2,5 , and $6 . \mathrm{X}$ signs mark the XBT locations and VLA indicates the vertical line array location.

over three distinct paths on whose intersection a vertical line array (VLA) was moored, as shown in Fig. 2. This paper reports the results obtained along the northwest (NW) path (event 2), along the northeast (NE) path (event 5) and between the NW and NE paths (event 6) (gray line in the figure). The path directed to the NW, parallel to the continental platform, is approximately range independent, while the NE path, oriented towards the coastline, is range dependent with water depths varying from $120 \mathrm{~m}$ at the VLA location to $70 \mathrm{~m}$ at the path end. In between these two paths, the environment is progressively and slowly changing from range independent to range dependent when going from NW to NE. As an overview of the technical aspects involved in the experiment, it can be referred that acoustic signals were transmitted with an acoustic transducer suspended from the research vessel NRP D. Carlos I, a Portuguese Navy oceanographic research ship managed by Instituto Hidrográfico (IH), and received on a 16-hydrophone 4-m-spacing VLA. The acoustic aperture of the VLA was located between the nominal depths of 30 and $90 \mathrm{~m}$ in a 120-m-depth water column. The acoustic signals received in the VLA were transmitted via an RF link to onboard ship, processed, monitored, and stored. The acoustic portion of the VLA was hanging from the sea surface and attached through a 70-m-long umbilical to the radio buoy that was itself bottom moored. Therefore, the VLA was navigating within a radius of approximately $70 \mathrm{~m}$ around the surface buoy mooring and moving up and down with surface motion and tides. This will be shown to be an important experimental detail during the analysis and validation of the acoustic data.

\section{A. Source-receiver geometry and events}

Figure 2 also shows the experimental site bathymetry along the acoustic paths together with the source ship tracks during these two events. At the beginning of event 2, the source ship started close to the VLA and then went off at low speed up to a range of approximately $5.5 \mathrm{~km}$ to the NW, transmitting precoded linear frequency modulated (LFM) signals. During event 5 the source ship approached the VLA from the NE along the range-dependent path while transmitting a pseudorandom noise (PRN) sequence. During event 6 the acoustic source was recovered and the ship itself was used as noise source. As seen on Fig. 2, during event 6, the ship started its run close to the VLA, went off to the NE leg for about $3.2 \mathrm{~km}$, and then performed a sharp turn to the

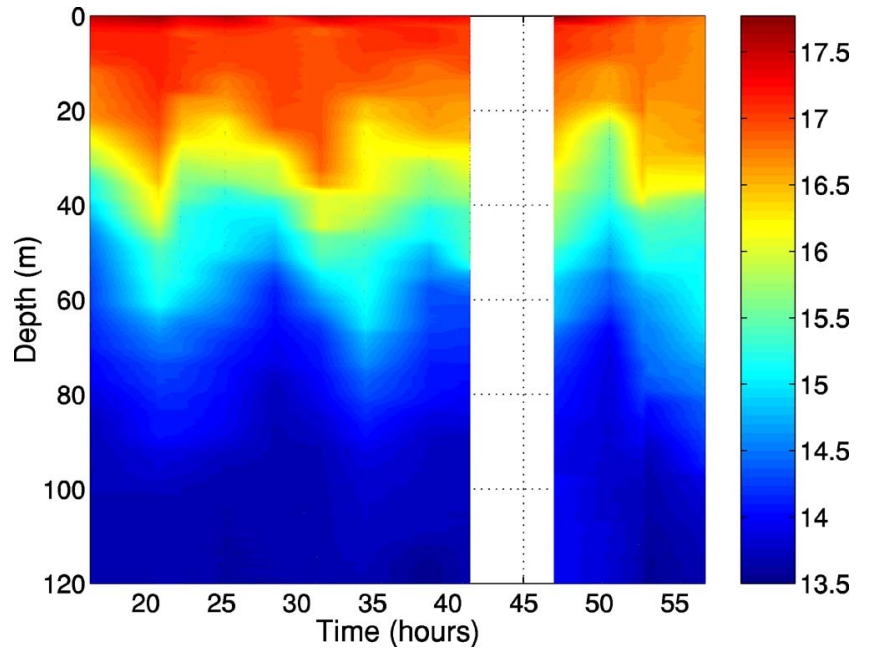

FIG. 3. (Color online) XBT cast temperature profiles.

west along a circular pattern around the VLA (gray line). The ship then repeated this pattern twice at VLA ranges of 2.2 and $1.2 \mathrm{~km}$, closing to the VLA. During event 6 the research vessel NRP D. Carlos I was steaming at her maximum speed of $10 \mathrm{kn}$, in an attempt to generate as much noise as possible.

\section{B. Environmental data measurements}

Environmental data include direct measurements performed during the cruise, like water column temperature and bathymetry along runs, as well as archival data with geological information of the area. Water column temperature was gathered from XBTs and a thermistor chain collocated with the VLA. XBTs were sparse in time, approximately one every $3 \mathrm{~h}$ in order to capture tidal evolution, and spatially distributed throughout the experiment site. See Fig. 2 for XBT location and Fig. 3 for the recorded temperature profiles. Thermistor chain recordings provide a high time resolution but a few observation samples in depth and at a single spatial location. The VLA has 8 temperature sensors at 8-m spacing. The first sensor is located $3 \mathrm{~m}$ below the shallowest pressure gauge that was recording a mean depth of $30 \mathrm{~m}$. So, temperature was recorded at approximate depths of 33, 41, 49, $57,65,73,81$, and $89 \mathrm{~m}$, depending on the precision of the pressure gauge sensor and tilt of the VLA. The temperature field is shown in Fig. 4(a)-4(c) for events 2, 5, and 6, respectively. All three events are of relatively short durations (between $45 \mathrm{~min}$ and $1 \mathrm{~h} 30 \mathrm{~min}$ ), which makes temperature recordings almost featureless. In all cases temperature is varying between 14 and 16.5 degrees Celsius with, in some cases, a few thermocline oscillations at the minimum observed depth of 30-35 m. Whether those temperature oscillations are due to effective thermocline changes or to VLA vertical movements is unknown.

The acoustic transmission tracks were surveyed during a previous sea trial in 1999 with both a sidescan sonar and a light seismic Sparker system. During INTIFANTE'00 a second sidescan sonar survey was performed. The overall conclusion is that the NW range-independent track used during event 2 has a mean depth of $119 \mathrm{~m}$ with a variation of $\pm 3 \mathrm{~m}$ after tide correction, while the bottom is composed of fine 
(a)

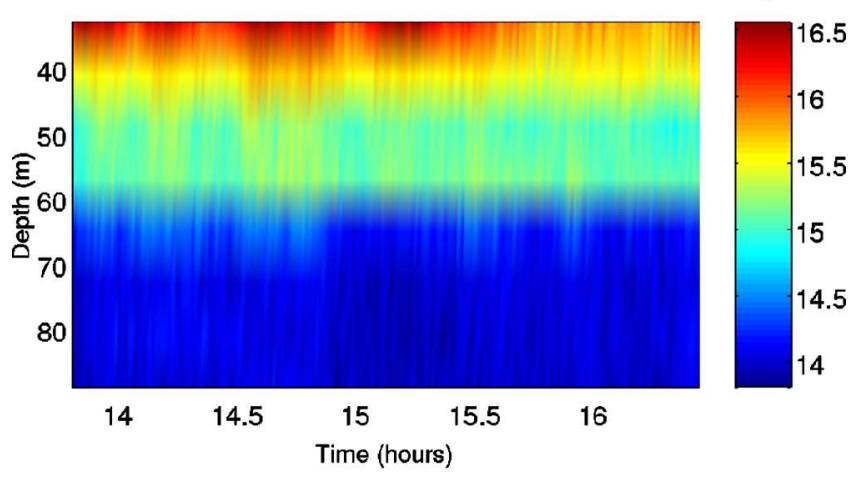

(b)

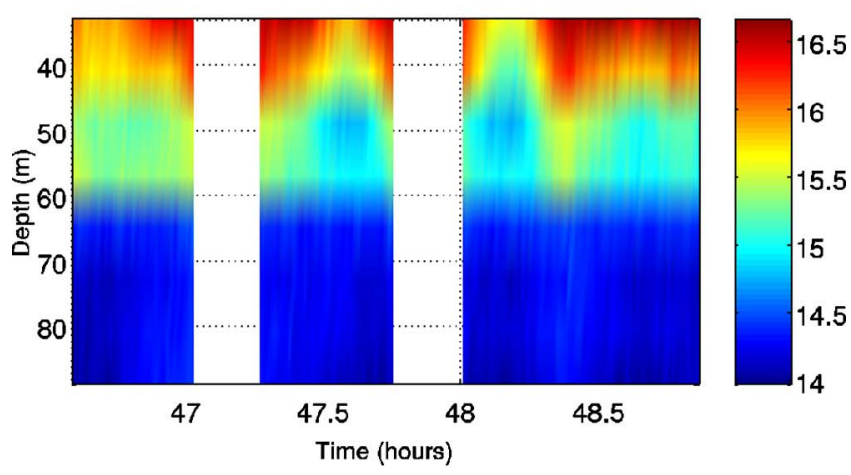

(c)

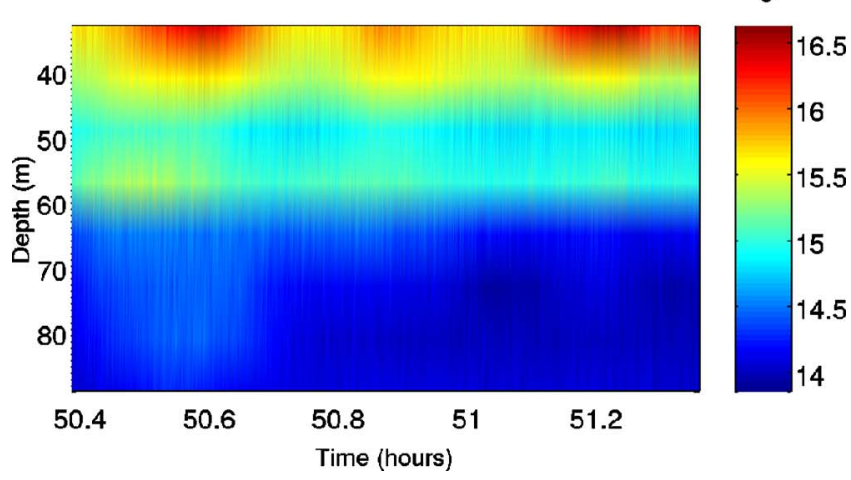

FIG. 4. (Color online) Temperature field recorded at the VLA during event 2(a), event 5(b), and event 6(c).

sand with a sediment layer of variable thickness between 4 and $6 \mathrm{~m}$, in the first $6 \mathrm{~km}$ from the VLA. The NE leg has highly variable bottom properties with mud and hard rock patches.

\section{Experiment geometry}

Source-receiver range is calculated from the known VLA mooring position and the ship GPS log and is superimposed with the estimated source-range results (see the figures of Sec. V). An additional source of information is the recording of ship speed and bearing during event 6 , as shown in Fig. 5, where it can be seen that the ship (acting as noise source in this event) maintained a mean speed of approximately $9 \mathrm{kn}$ with several speed drops during sharp turns. (a)

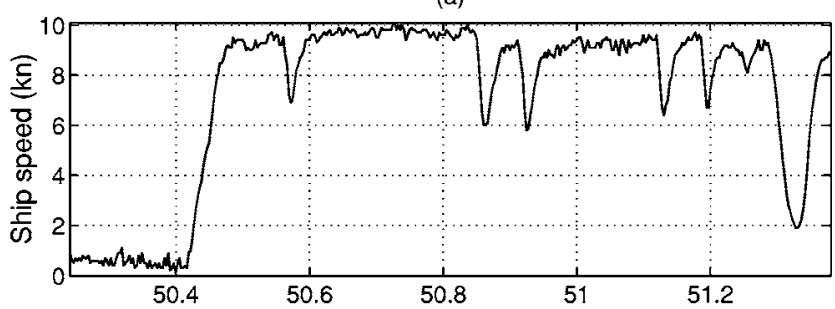

(b)

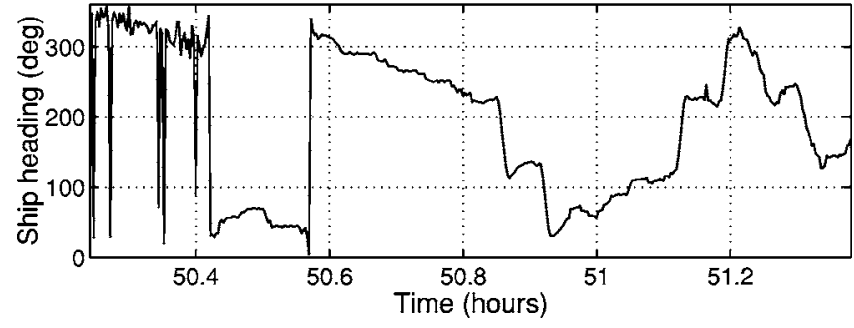

FIG. 5. GPS estimated ship speed (a) and ship heading (b) during event 6.

Unfortunately, the depth sensor collocated with the sound source failed to work during the whole experiment, so only information from cable scope and source depth variation versus tow cable length for various tow speeds was available to estimate source depth during the various runs.

\section{ENVIRONMENTAL MODELING}

An important first step in tomographic inversion is the choice of an environmental model able to represent the mean characteristics of the media where the signal is propagating. Such model will be called the baseline model and generally includes the available a priori information for the problem at hand. Besides the column temperature measurements and the sidescan sonar survey described in the previous section, there were no other in situ geological observations (e.g., cores) that could be used as additional background information, specifically for setting up the model bottom characteristics. Thus, it was decided to adopt a very generic baseline environmental model consisting of an ocean layer overlying a sediment and a bottom half-space assumed to be range independent, as shown in Fig. 6. The geoacoustic properties were drawn from generic geological knowledge of the area where it was assumed that the NW range-independent track had a quite regular bottom, covered by fine sand. Since event 5 is made along the NE track, a range-dependent version of the environmental model shown in Fig. 6 was used, where the bathymetry is simulated by a uniform bottom slope with a water depth varying from $70 \mathrm{~m}$ at the source location to $119 \mathrm{~m}$ at the VLA location. Event 6 takes place between the NW and NE tracks, where the latter is range dependent. In this case a range-independent model was used and therefore some degree of environmental model mismatch is anticipated.

Another important problem when inverting acoustic data is the difficulty associated with the representation of the sound-speed field in time, depth, and range by a finite set of invariant parameters. The solution for this problem is known as data regularization, and it consists of the expansion of the temperature, or equivalently, the sound-speed field, if the sa- 


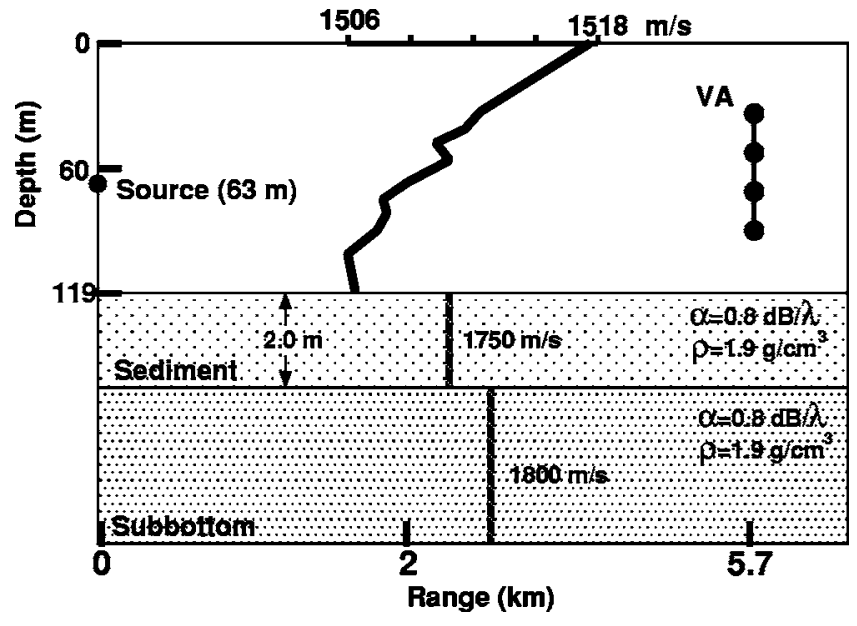

FIG. 6. Baseline environmental model for the range-independent propagation scenario of event 2 .

linity profile in the area is known or can be represented with some degree of accuracy by a constant value over depth, on a basis of functions representative of the data set to be estimated. Since the inverse problem is constrained by the basis functions used, it is of paramount importance to determine a suitable function basis for each data set. It is well known that regularization is a powerful method that leads to a faster convergence and a higher uniqueness of the optimal solution, since prior information already contained in the set of basis functions allows one to start the search closer to the optimal solution than if no such information was available. A wellknown method for obtaining a suitable basis function is to calculate the empirical orthogonal functions (EOF) as the eigenfunctions of the data correlation matrix. Assuming that the temperature field is stationary in range, this method requires a time series representative of the data under observation taken in the location and at the time of the experiment. In other words, in order to obtain a tomographic image of the temperature data one needs to already have an observation of those data. In many situations, and also in passive tomography, that is a strong a priori requirement that is not always fulfilled. Alternatively, it has been shown that a suitable set of eigenfunctions can be formed from the hydrodynamical normal modes (HNM) of oscillation of the temperature field. ${ }^{25}$ In that case only a mean temperature profile from archival data is necessary.

In our case, since a full set of observations was readily available, the EOF method was used for parametrization of the ocean temperature. The EOFs were obtained using a singular value decomposition (SVD) of a data matrix $\mathbf{C}$ with columns

$$
\underline{C}_{i}=\underline{T}_{i}-\underline{\bar{T}}
$$

where $\underline{T}_{i}$ are the real profiles available, and $\bar{T}$ is the average profile. The SVD is known to be given by

$$
\mathbf{C}=\mathbf{U D V} \text {, }
$$

where $\mathbf{D}$ is a diagonal matrix with the singular values, and $\mathbf{U}$ is a matrix with orthogonal columns, which are used as the EOFs. The temperature profile is obtained by
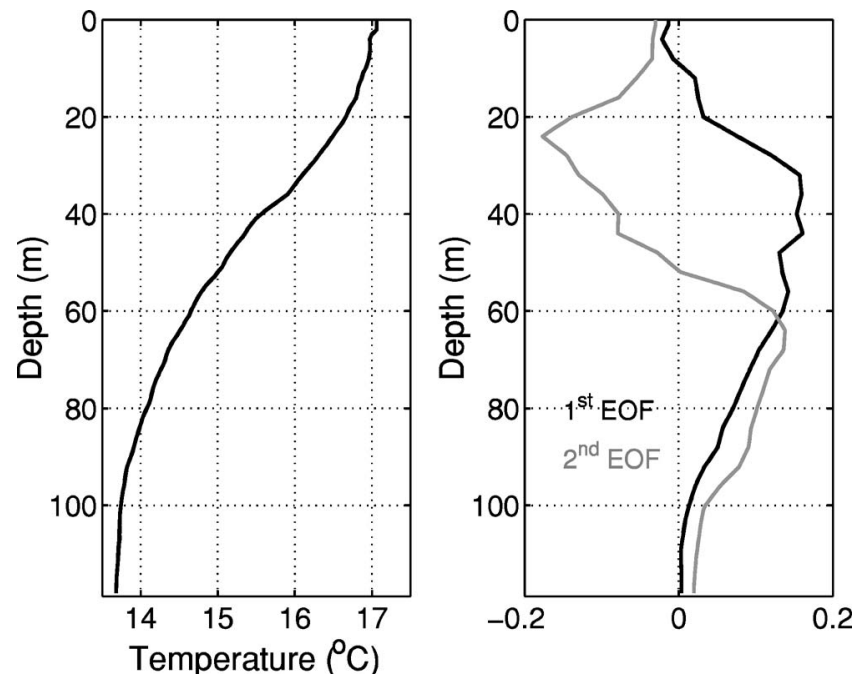

FIG. 7. XBT-based data used for temperature estimation: mean temperature profile (left) and first two empirical orthonormal functions (EOFs) (right).

$$
\underline{T_{\mathrm{EOF}}}=\underline{\bar{T}}+\sum_{n=1}^{N} \alpha_{n} \underline{U}_{n},
$$

where $N$ is the number of EOFs to be combined, judged to accurately represent the temperature field for the problem in hand. Generally, a criterion based on the total energy contained on the first $N$ EOFs is used. Experimental results have shown that usually the first 2 or 3 EOFs are enough to achieve a high degree of accuracy. The 14 temperature profiles obtained from the XBT measurements (see Ref. 24) served as a database for the computation of the EOFs.

The criterion used to select the number of relevant EOFs for the available data was

$$
\hat{N}=\min _{N}\left\{\frac{\sum_{n=1}^{N} \lambda_{n}^{2}}{\sum_{m=1}^{M} \lambda_{m}^{2}}>0.8\right\},
$$

where the $\lambda_{n}$ are the singular values obtained by the SVD, $M$ is the total number of singular values, provided that $\lambda_{1}$ $\geqslant \lambda_{2} \geqslant \cdots \geqslant \lambda_{M}$. For this data set criteria (4) yielded $\mathrm{N}=2$, i.e., the first two EOFs are judged sufficient to model the sound speed with enough accuracy (see Fig. 7). The coefficients $\alpha_{n}$, which are the coefficients of the linear combination of EOFs, are now part of the search space, i.e., they are searched as free parameters.

\section{FOCALIZATION: A METHOD FOR GLOBAL INVERSION}

Multiple environmental and geometrical parameter optimization is often a computationally cumbersome task. The optimization hypersurface may be very irregular, leading to a severe ill-conditioned problem with a large number of local extrema. When dealing with real data, the inherent model mismatch and the presence of noise create a situation where there is no assurance of existence of an optimum solution in coincidence with (or even close) to the true model parameters. The first approach to the problem is to try to get as much a priori information as possible from the environmen- 
tal parameters into the baseline model, in order to set them fixed and close to the true parameters, so the search is only done on a few unknown parameters. In practice, it is well known that setting fixed parameters in the model creates severe mismatches with real data that cannot be overcome by the search parameters, leading to poor fit situations and strongly biased estimates. An alternative is a technique proposed by Collins et al., ${ }^{21}$ known as focalization, where a number of a priori known model parameters, are allowed to be adjusted during the search process in order to compensate for the data-model misfit and possible measurement errors. Generally, a priori known parameters have a smaller degree of variation than the unknown parameters. This technique provides a high degree of adaptivity, a higher model fit, and a better-conditioned convergence to the true parameter values. Examples are given in recent publications by the authors. $^{18,20,26,27}$

The optimization technique for reducing the number of forward computations was based on a genetic algorithm (GA). Principles of GA are now well known in the underwater acoustic community and elsewhere, and various strategies have been widely used in practice with positive results. GA parameter setting may have a strong influence on convergence to the solution and hence on the final result. These parameters have been set differently for each case and are explained below. The GA implementation used in this study was proposed in Ref. 28. In particular, a new scheme that was found to drastically optimize the search is to use the final solution at a given time period in the initialization of the solving procedure of the next time period.

Let us assume that at time $t_{i}$ the best individual of the last population is $b\left(t_{i}\right)$. The GA is initialized at time $t_{i+1}$ such that $30 \%$ of the individuals of the initial population are uniformly distributed within a $10 \%$ variation interval of the coordinates of $b\left(t_{i}\right)$. The other $70 \%$ are randomly distributed in the whole search space, in order to maintain a high degree of diversity. With this procedure the number of iterations has been decreased at each time period except for the first one. In practice it is verified that the model fit drops at the beginning of each time period when compared with its value at the end of the previous time period, which denotes that the data have changed and that the model is catching up through the adjustment of the parameters. However, after that initial fitness drop, rapid convergence to their "right" values, or at least to those giving the highest fit, is obtained. The objective function used in this study was based on the incoherent Bartlett processor in a frequency band selected according to the sound source response function. In order to be more specific, let us recall the Bartlett power function

$$
B\left(\theta, t_{n}\right)=\frac{1}{K} \sum_{k=1}^{K} \mathbf{w}\left(\omega_{k}, \theta\right)^{H} \hat{\mathbf{R}}_{Y}\left(\omega_{k}, t_{n}\right) \mathbf{w}\left(\omega_{k}, \theta\right),
$$

where $\hat{\mathbf{R}}_{Y}\left(\omega_{k}, n\right)$ is the data cross-correlation matrix estimate at frequency $\omega_{k}$ and at time interval $t_{n}, \mathbf{w}\left(\omega_{k}, \theta\right)$ is the model replica acoustic pressure at frequency $\omega_{k}$ and for search parameter $\theta, K$ is the number of frequencies, and ${ }^{H}$ indicates conjugate transpose. The number and values of the selected frequencies varies with each case and as a general rule it was a compromise between the degree of difficulty of the problem at hand and the computational load. The C-SNAP code ${ }^{29}$ was used as forward model.

\section{RESULTS}

In order to gain some insight into the processing of the ship noise data for the purpose of BOAT, it was decided to first test methods and algorithms with the active source data along both the NW range-independent and the NE rangedependent tracks, respectively, described in Secs. V A and V B. Since the ship noise data was obtained with a (fast) moving ship it was decided to analyze parts of data of these two events involving moving sources.

\section{A. Active data in a range-independent track: Event 2}

During event 2, a series of acoustic 170-600-Hz LFM sweeps was transmitted over a range-independent shallowwater waveguide, while the source was towed away from the VLA location. Results are shown in Fig. 8, and the following comments apply: the Bartlett power [given by (5)] is relatively high throughout the run, source range is well estimated, and all the other parameters are jointly estimated with credible values, including bottom properties. The temperature profiles are modeled by two EOFs whose coefficients show a smooth evolution through time, giving rise to a nicely stratified temperature estimate. Note that the environmental cross section being inverted for changes with time since the source is moving away from the VLA which, to some extent, may explain the observed high variability of non-timevarying parameters such as sediment and bottom sound speed as well as sediment thickness.

\section{B. Active data in a range-dependent track: Event 5}

During event 5 the sound source was emitting a pseudorandom noise (PRN) sequence in the band $150-1100 \mathrm{~Hz}$, supposed unknown at the receiver. The inversion results are shown in Fig. 9. This run is a good example on how the three indicators - source range, source depth, and Bartlett powercan be used to validate environmental model estimates. At the beginning of the run, until time 47.04, the Bartlett power varies between 0.4 and 0.8 , source range changes rapidly, most of the other parameters have highly variable values, and some are on, or near, the bounds of their search intervals. So, in this initial period, temperature estimates [plot $(\mathrm{k})$ ] cannot be considered as valid. At time 47.04, source range is suddenly estimated very close to the true values at $4-\mathrm{km}$ range and steadily follows the approaching of the source to the VLA up to time 48 at about $2-\mathrm{km}$ source range. During that interval most of the parameters, except the sediment thickness and the EOF coefficient $\alpha_{1}$, follow stable values well within their respective intervals and are therefore mostly credible. The first EOF coefficient suffers a strong, and to date unexplained, change at time 47.52 right in the middle of that smooth path. After time 48, when the source has reached the closest point of approach to the VLA, the model match is again suddenly lost with strong variations on all parameters: drop of the Bartlett power from 0.8 to 0.3 , a sudden range 
(a)

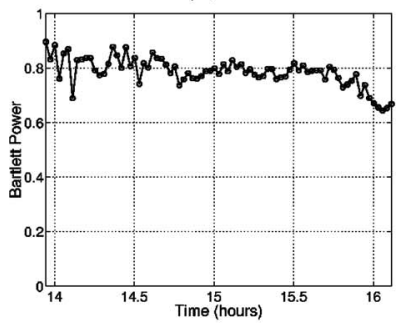

(d)

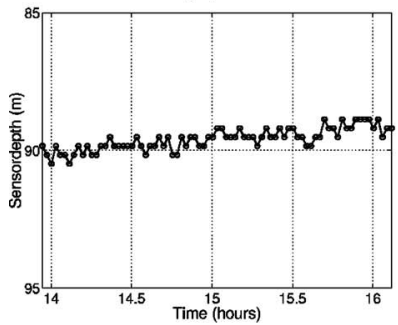

(g)

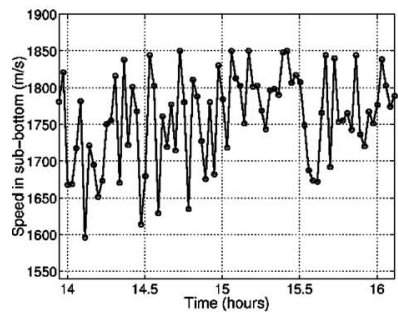

$(\mathrm{j})$

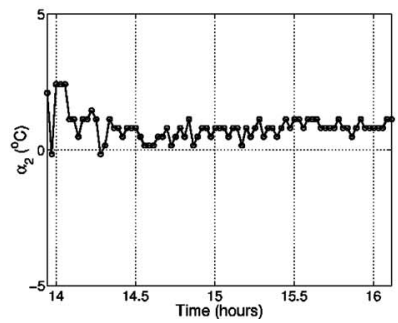

(b)

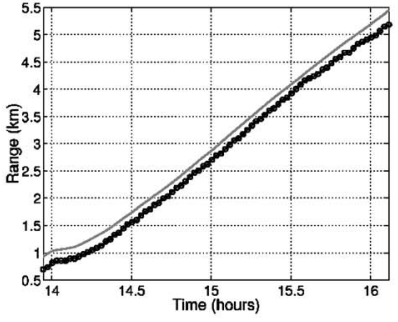

(c)

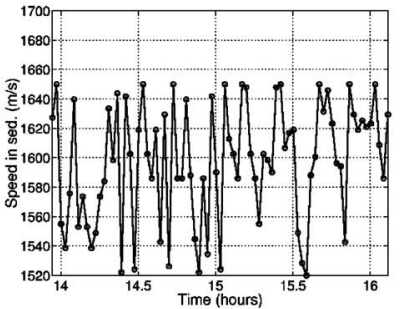

(h)

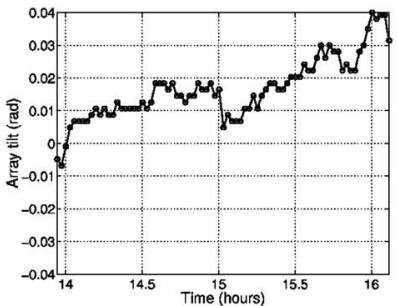

(k)

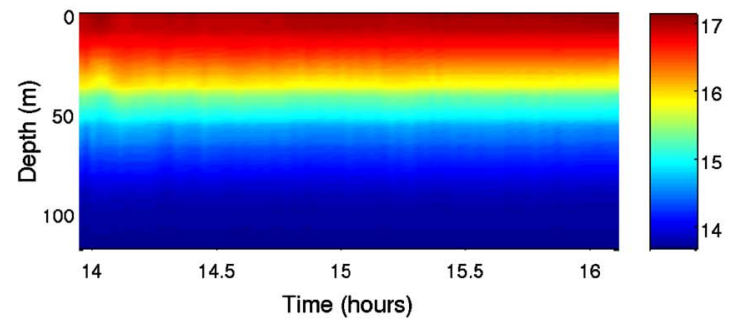

FIG. 8. (Color online) Focalization results for event 2: Bartlett power (a), source range (b), source depth (c), receiver depth (d), sediment compressional speed (e), sediment thickness (f), sub-bottom compressional speed (g), VLA tilt (h), EOF coefficient $\alpha_{1}$ (i), EOF coefficient $\alpha_{2}$ (j), and reconstructed temperature profiles $(\mathrm{k})$.

variation from 1 to $3.5 \mathrm{~km}$, and a drop of $10 \mathrm{~m}$ on source depth. After the work of Collins, ${ }^{21}$ and using an analogy with optics, the terms "focus" and "out of focus" are often used to designate these alternate model adjustments and misadjustments. It was found that time 48 coincides with the low-tide change producing a $1.5-\mathrm{m}$ rise on the array accompanied by strong variations of array tilt, as measured on the depth sensors and tiltmeters on the VLA [see Fig. 4.9 plots (a), (b), and (c) of Ref. 30]. There is the belief that during the tide change the array assumed a shape that was not taken into account by the baseline model. The model regains stability after 15 min with smooth parameter estimates and high Bartlett power values. Among all obtained values within validated intervals, source range and depth were clearly in agreement with the expected values; sound speed in the sediment and bottom are reasonably well estimated to have expected mean values of 1580 and $1700 \mathrm{~m} / \mathrm{s}$, respectively, with a higher uncertainty in the latter; and finally array depth and array tilt are in good agreement with the pressure and tilt sensors collocated with the VLA. After focalization the water temperature was reconstructed — plot $(\mathrm{k})$ — showing a highly perturbed estimate due to successive focus and loss of focus through time. Also in this case the environment cross section is changing along time, possibly even more than in event 2 , since the environment is range dependent with patches of rock and mud as reported in geological charts of the area; therefore, an even higher variability of bottom parameters is anticipated.

\section{Passive data in a partially range-dependent track: Event 6}

The real challenge comes when addressing the problem of tomographic inversion using ship noise data, i.e., a real unknown and stochastic source signal at unknown location and moving in a poorly known environment. This chapter addresses this problem using as example the data gathered 
(a)

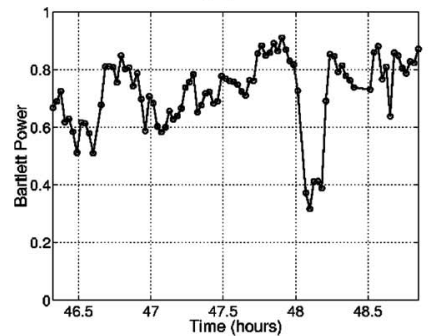

(d)

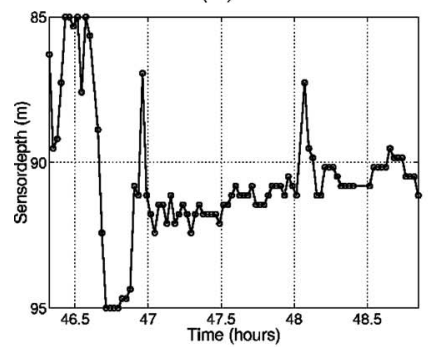

(g)

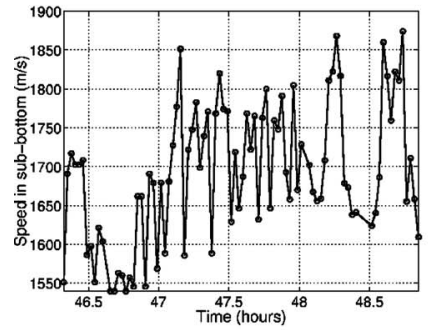

(j)

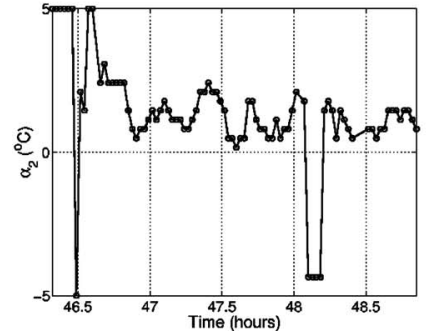

(b)

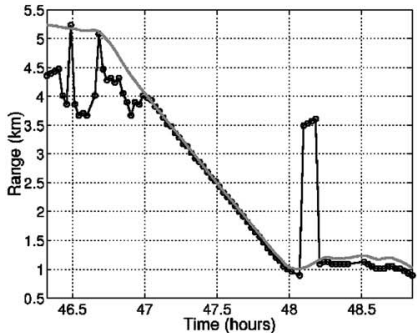

(c)

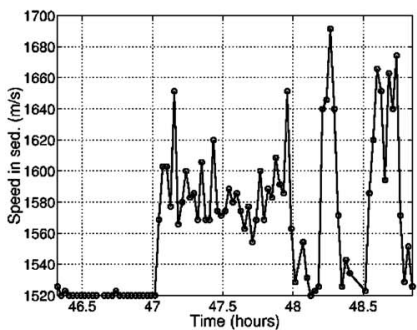

(h)

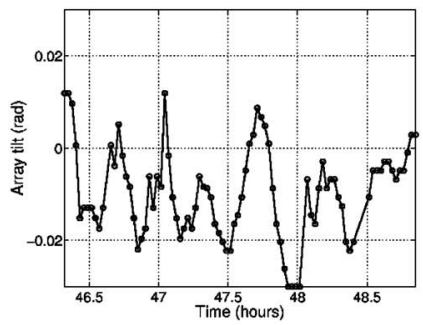

(k)

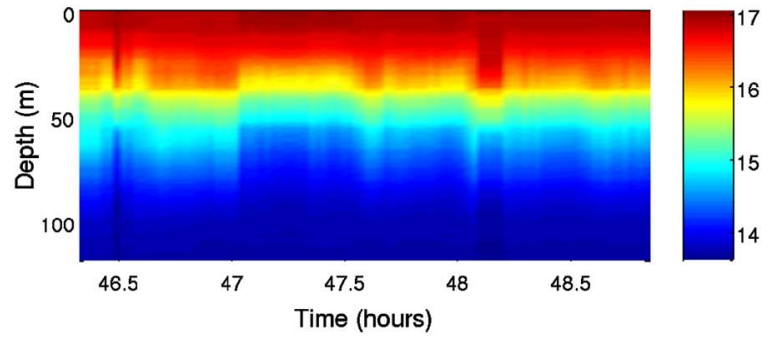

FIG. 9. (Color online) Focalization results for event 5: Bartlett power (a), source range (b), source depth (c), receiver depth (d), sediment compressional speed (e), sediment thickness (f), sub-bottom compressional speed (g), VLA tilt (h), EOF coefficient $\alpha_{1}$ (i), EOF coefficient $\alpha_{2}$ (j), and reconstructed temperature profiles (k).

during event 6, when the acoustic source was replaced by the NRP D. Carlos I herself as noise signal generator for tomographic inversion purposes.

\section{Ship-radiated noise}

Research vessel NRP D. Carlos I is a 2800-ton relatively recent ship, built in 1989, whose primary purpose was acoustic surveillance when it served under the U.S. flag. She has an overall length of $68 \mathrm{~m}$ and a beam of $13 \mathrm{~m}$. Her main propulsion system is formed by two diesel-electric engines developing $800 \mathrm{HP}$ attaining a maximum speed of $11 \mathrm{kn}$. According to her characteristics NRP D. Carlos I can be considered as an acoustically quiet ship. Hence, her use for the purpose of passive tomography can be considered as providing conservative results when compared with full-length cargo ships or tankers traveling at cruising speed. In order to maximize the probabilities of successful inversion and get close to the cruising speeds of "normal" ship traffic, NRP D. Carlos was set to steam at her full speed along a series of three concentric portions of circle as shown in Fig. 2 (gray line) with ship's speed and heading as shown in Fig. 5, plots (a) and (b), respectively. As an example, Fig. 10 shows a time-frequency plot of the relative power spectrum received on hydrophone 8 at $60-\mathrm{m}$ depth (a), and a mean power spectrum over the whole event (b). There are clearly a few characteristic frequencies emerging from the background noise between 250 and 260 and a strong single tone at $359 \mathrm{~Hz}$. There is also a colored noise spectra in the band 500 to $700 \mathrm{~Hz}$ with, however, a much lower power.

\section{Environmental model}

During this test several difficulties are added to the problem, when compared to the previously analyzed data set obtained in events 2 and 5: (i) the source is moving fast; (ii) the 
(a)

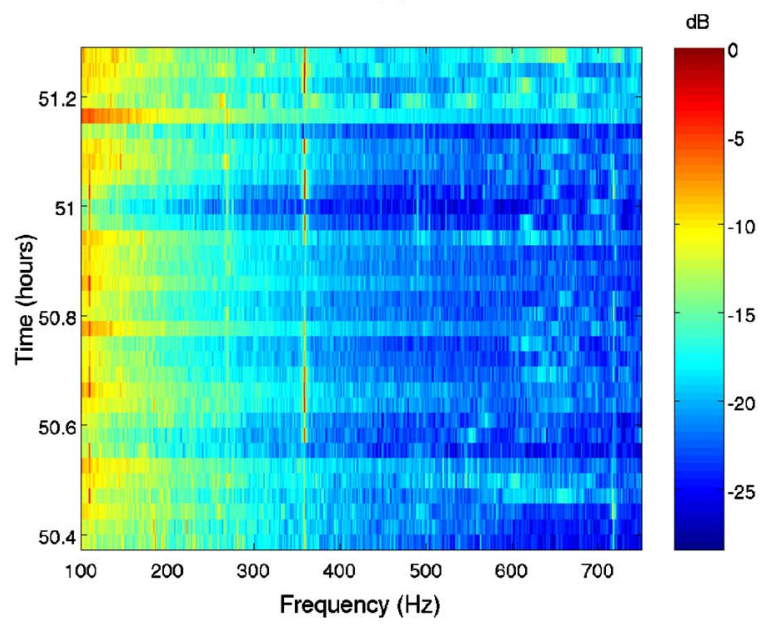

(b)

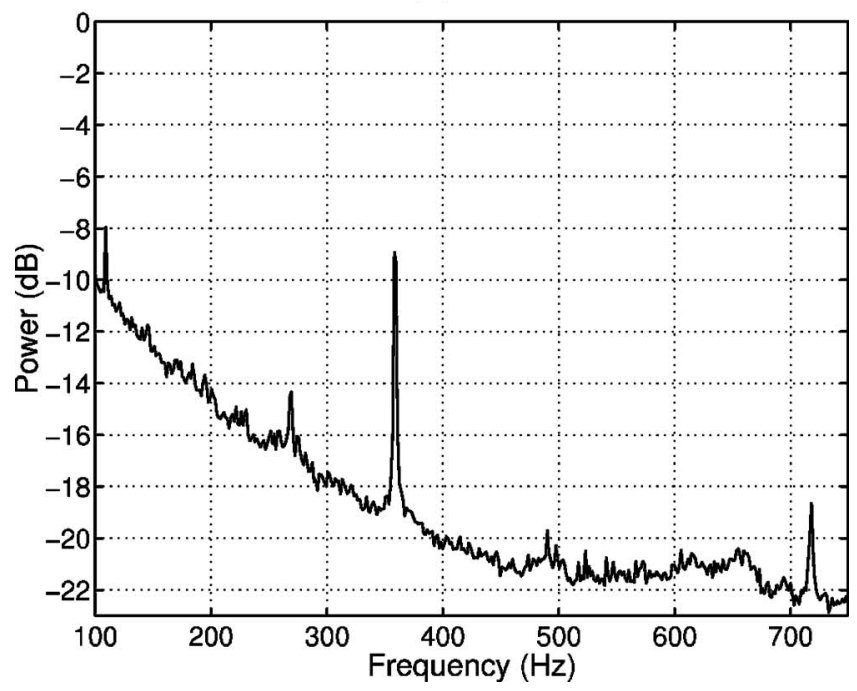

FIG. 10. (Color online) NRP D. Carlos I ship radiated noise received on hydrophone 8 , relative power scale on a time-frequency plot (a) and mean power spectrum (b).

environment is a mixture of range dependent and range independent; and (iii) the source signal is ship noise with unknown and presumably time-varying characteristics. On top of those difficulties, the actual processing adds also a further problem which is that it is not possible to decide during the processing to switch between range-independent and rangedependent environmental models. In theory, a rangedependent model is also applicable to the range-independent case, allowing water depth at the source end to change along the ship track. Due to the well-known source range versus water depth inter-relation and parameter hierarchy, it is impossible, or at least extremely difficult, to simultaneously estimate source range and water depth as well as other low dependence parameters. In this analysis it was decided to use a range-independent model for reducing the computation and inversion burden. As will be seen in the following section, the usage of a range-independent model, even in a slightly range-dependent environment, will add a significant source range mismatch, at some well-defined points during the processing.

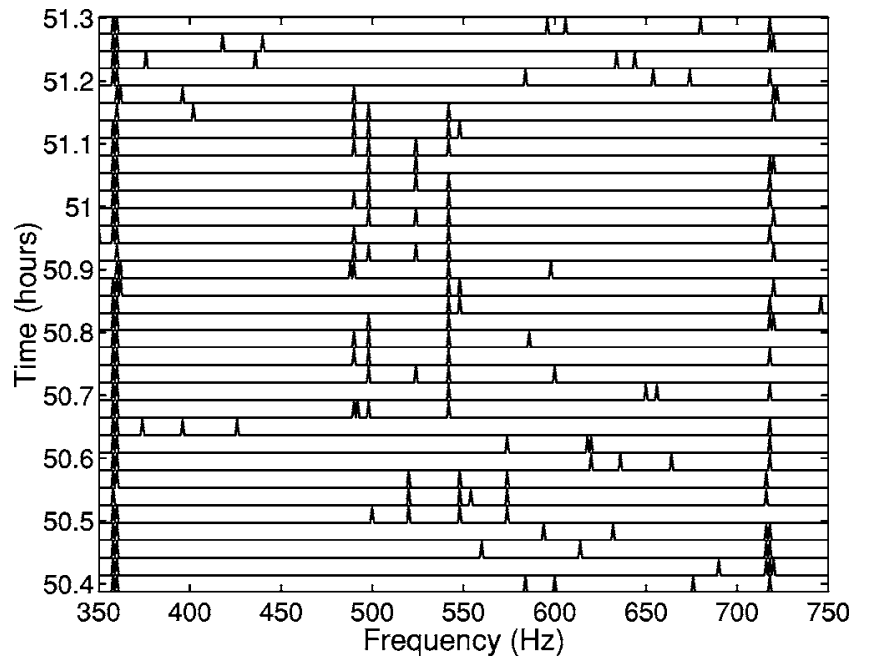

FIG. 11. INTIFANTE'00 sea trial, event 6 frequency selection: 1 - selection and 0 - no selection based on contiguous snapshot mean signal coherence along time [Eq. (6)].

\section{Inversion results with a coherence-based frequency selection}

The inversion methodology was based on a three-step procedure: (i) preliminary search of the outstanding frequencies in a given time slot; (ii) the usual parameter focalization, based on an incoherent broadband Bartlett processor; a C-SNAP (Ref. 29) forward acoustic model and a GA based optimization and (iii) inversion result validation based on model fitness and coherent source range and depth estimates through time. There are a number of possibilities for implementing the frequency selection procedure for the first step. A first attempt using a simple periodogram based spectral estimator for frequency selection was shown in Ref. 31 and a similar estimator coupled with an amplitude estimator for frequency selection and weighting in Ref. 23. In the present paper a coherence-based approach is proposed, where the time coherence of a given frequency $\omega$ is evaluated according to

$$
\Gamma(\omega)=\frac{1}{N-1} \sum_{n=1}^{N-1} \frac{\mathbf{Y}^{H}\left(\omega, t_{n+1}\right) \mathbf{Y}\left(\omega, t_{n}\right)}{\left\|\mathbf{Y}\left(\omega, t_{n+1}\right)\right\|\left\|\mathbf{Y}\left(\omega, t_{n}\right)\right\|} \geqslant \gamma_{0},
$$

where $\mathbf{Y}\left(\omega, t_{n}\right)$ is an $L$-dimensional complex vector with the $L$ array sensor output at frequency $\omega$ in time snapshot $t_{n}, N$ is the total number of time snapshots in a given window, and $\gamma_{0}$ is a constant detection threshold depending on the actual SNR and signal/ambient noise level. The idea is that if a signal is present in a given frequency bin, a slow change of the channel structure would make this signal maintain its coherence from one snapshot to the other while there is a good chance that ambient noise will have a lower time coherence. For the data of event $6,16 \mathrm{~s}$ of data were divided into 0.5-s-duration snapshots, thus giving a frequency resolution of $2 \mathrm{~Hz}$, and a number of snapshots $N=32$. The result of applying (6) in the frequency band 350 to $750 \mathrm{~Hz}$ gave the results shown in Fig. 11. This figure shows which bins were selected for processing - the third axis is 1 upon selection, and 0 otherwise. Note that there are a couple of frequencies almost constantly present through- 
(a)

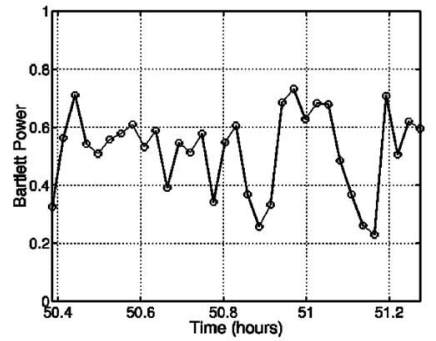

(d)

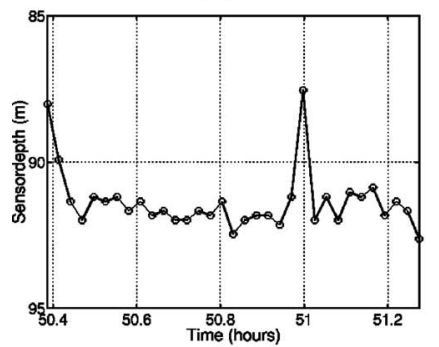

(g)

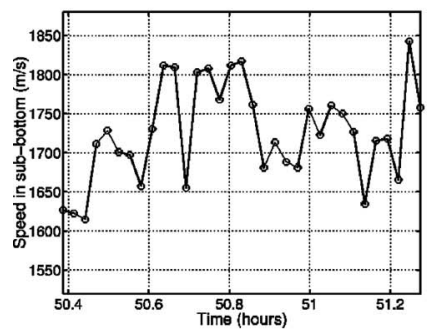

(j)

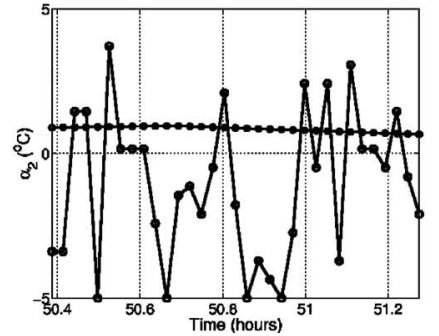

(b)

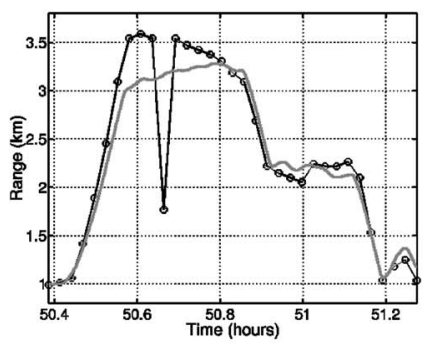

(e)

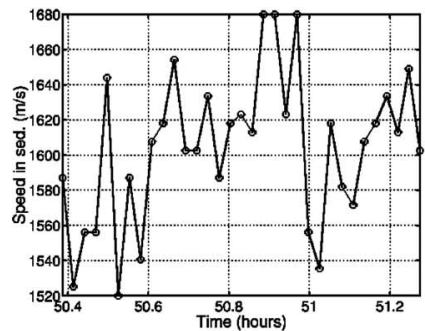

(h)

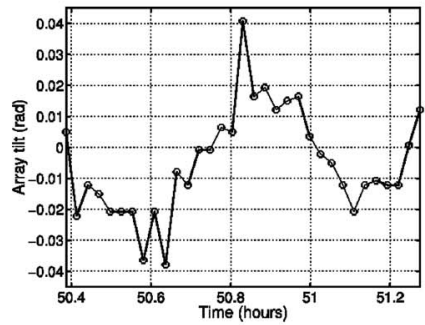

$(\mathrm{k})$

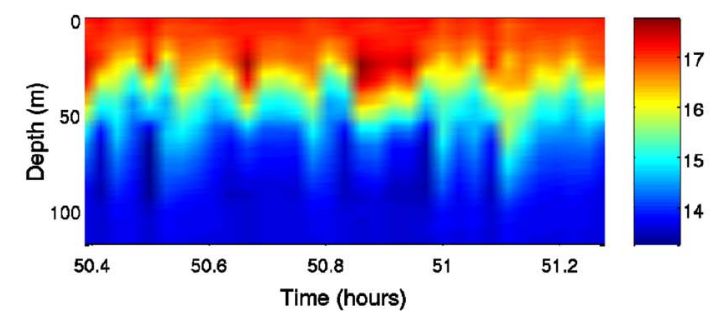

FIG. 12. (Color online) Focalization results for event 6: Bartlett power (a), source range (b) [the continuous line is the GPS measured source-receiver range], source depth (c), receiver depth (d), sediment compressional speed (e), sediment thickness (f), sub-bottom compressional speed (g), VLA tilt (h), EOF coefficient $\alpha_{1}$ (i), EOF coefficient $\alpha_{2}$ (j) [filled dotted lines are the XBT measured data projected onto the respective EOFs], and reconstructed temperature (k).

out the run at 359 and $719 \mathrm{~Hz}$, where one might be a harmonic of the other, as well as other high coherence bins with some persistence at 490, 498, and $542 \mathrm{~Hz}$. Actually, instead of setting $\gamma_{0}$, and in order to limit the computational complexity of the problem, a fixed number of frequency bins were selected at each time slot according to the maximum values of (6). The inversion results are shown in Fig. 12: from (a) to (j) are individual parameter estimates, while plot $(\mathrm{k})$ shows the water column temperature reconstruction based on the EOF linear combination of parameter estimates (i) and (j). Plot (b) shows the estimated source range together with the GPS measured source range (continuous line). At first glance from the model fit indicator (Bartlett power) the result is poor, since it is always below 0.8 ; however, source range, which is one of the leading parameters, shows values in coincidence with the GPS measured source range while source depth is highly incoherent within the 0-6-m depth inter$\mathrm{val}$; and finally the reconstructed temperature [plot $(\mathrm{k})$ ] appears to be too variable for such a small time interval (slightly over $45 \mathrm{~min}$ ). Looking more in detail, and comparing plot (a) of Fig. 5 with plots (a)-(c) of Fig. 12, the following conclusions can be drawn: (i) for $50.42 \leqslant$ time $\leqslant 50.57$, ship speed increases steeply to $9 \mathrm{kn}$, while heading off from the VLA. Range variation is about $4.6 \mathrm{~m} / \mathrm{s}$, which may cause a violation of the stationary assumption during the averaging time. Source range estimation error progressively increases as the ship reaches the longest range point and then remains high during part of the first loop trajectory at an approximate constant range of $3.2 \mathrm{~km}$; this erroneous source range estimate is almost certainly due to the environmental water depth mismatch in this portion of the track both along the NE track (event 5) and part of the trajectory to the NW track (see Fig.2). 
After the end of the first loop the estimated source range perfectly matches the GPS curve. As mentioned above, source depth [plot (c)] is highly variable in the interval $0-6-\mathrm{m}$ depth, which is understood to be due to the nature of the emitted signal (ship noise) radiated from a structure extending below but also on or above the sea surface. Receiving array estimates shown in plots (d) and (h) for array depth and tilt, respectively, are in agreement with the expected values. Notice the interesting behavior of array tilt that varies from -0.03 to +0.03 almost linearly during the side-looking view $45 \mathrm{deg}$ change when running from the NE to the NW leg and then back to the NE leg at the end of the run. Concerning the seafloor properties, one can say that their estimates are confined to relatively short time intervals at periods when the vessel is steaming at her maximum speed, which somehow justifies their high variability as seen on plots (e) to (g). EOF coefficients $\alpha_{1}$ and $\alpha_{2}$ are shown in plots (i) and (j), respectively. The estimated values are highly variable within the search interval, which is believed to be due to the highly variable and extremely low number of frequencies available in the ship-radiated noise spectrum, associated with ship's acceleration and deceleration during maneuvering. As a final comment on Fig. 12, the reconstructed water temperature-plot (k) - suffers both from poor estimation and ship variability. A different way of looking at the results is to plot histograms of the estimates as shown in Fig. 13, where it can be seen that $\alpha_{1}$ 's most frequent estimate is -3 and that of $\alpha_{2}$ is 0 , which are values compatible with those measured with the XBT during that period of time [filled dots curve on plots (i) and (j)].

\section{DISCUSSION AND FINAL CONCLUSIONS}

OAT is an appealing technique for remote monitoring of the ocean volume. One of the basic principles of OAT is that both source(s) and receiver(s) are under control of the experimentalist, that is, the emitted source signal and the source-receiver geometry is known (with some degree of precision) at all times during the observation window. In passive tomography the control of the source is relaxed, in order to be able to take advantage of possible sources of opportunity passing within acoustic range from the receiv$\mathrm{er}(\mathrm{s})$. Although passive tomography is very appealing for the ease of application, its practical implementation is extremely challenging and its feasibility remains to be proved.

This paper shows the tomographic inversion results obtained on a dataset obtained during the INTIFANTE'00 sea trial aiming at proving the feasibility of passive tomography. The results are presented in increasing order of difficulty for applying BOAT with a deterministic moving source in a range-independent environment, a pseudorandom source in a range-dependent environment, and finally using the noise radiated by a fast moving ship in a partially range-dependent environment. The challenge is represented by the fact that during the various phases of the processing the a priori knowledge about the source is progressively relaxed, leading to a situation close to that encountered in plain passive tomography. (a)

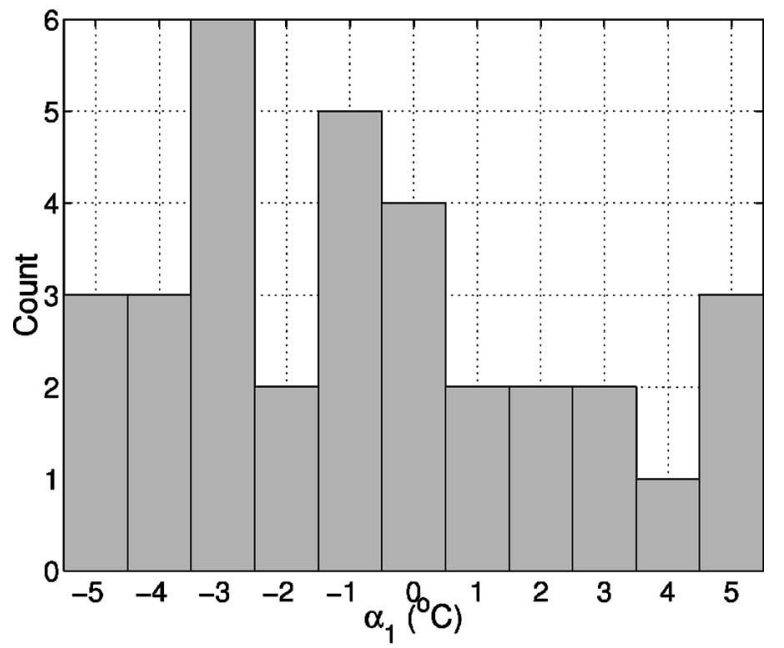

(b)

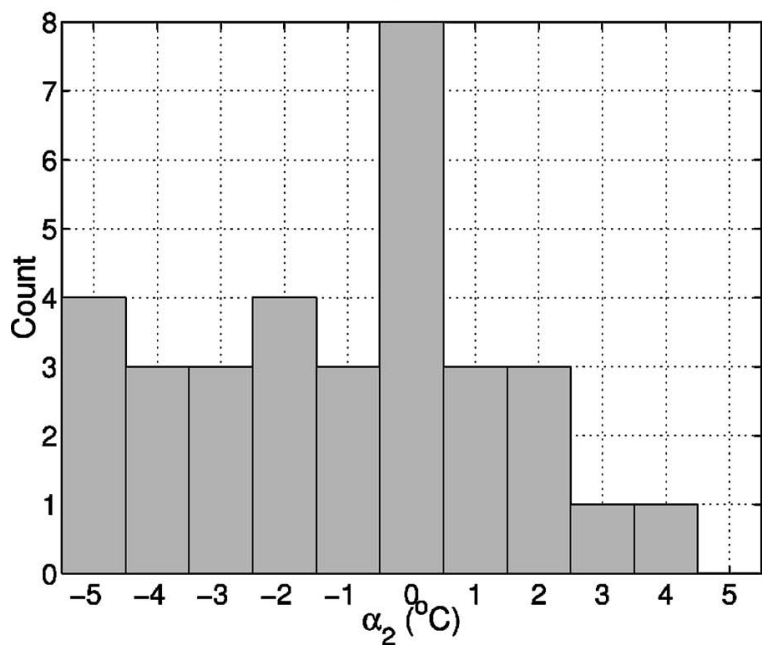

FIG. 13. INTIFANTE'00 sea trial, event 6: histograms of the EOF coefficients estimates for $\alpha_{1}$ (a) and $\alpha_{2}$ (b).

In a first data set it is proved that a moving source at an unknown location emitting a deterministic unknown signal over a range-independent environment can be used for ocean tomography adapting to the environment in time. Estimates of the various environmental and geometric parameters are consistent with the expected values. Focalization was demonstrated to represent the tool of choice, accounting for the unknown geometric and environmental parameters. In a second data set a moving source at an unknown location was emitting a PRN sequence used for determining the soundspeed structure as well as other geometrical and geoacoustic parameters over a range-dependent environment. The results were also consistent with expectations and demonstrated that the employed methods can operate, although with increasing difficulty, in range-dependent environments with both deterministic and pseudorandom noise source signals. Most importantly, it was shown that high-ranking parameters such as source range and depth together with the Bartlett fit could be used as indicators of the estimates' validity throughout the run. Finally, a third data set, where the controlled sound source was replaced by the ship itself moving at high speed in a series of concentric loops around the receiving array, 
showed that during some "clear" periods credible environmental estimates were obtained with the source ship correctly positioned and presumed correct environmental parameters.

During the analysis of this data set severe concerns were raised regarding the proper account for the vertical line array (VLA) geometry into the processor. Based on real-time measurements of the array tilt and sensor depth, the VLA was suspected to be responsible for several data mismatches during the inversion process. Those mismatches were seen to happen in conjunction with tidal movements at the array location. Another concern relates to the useful bandwidth of the radiated ship noise for environmental inversion. Although that concern was partially mitigated by the frequency selection based on the signal's short time coherence, a doubt remains whether real cargo ships at cruising speeds do radiate enough bandwidth sufficiently loud for the purpose of BOAT. The authors believe that these results demonstrate that blind ocean acoustic tomography is feasible and worth further development.

\section{ACKNOWLEDGMENTS}

The authors would like to thank the Instituto Hidrográfico, Lisbon (Portugal) for the organization of the INTIFANTE'00 sea trial and E. Muzi from NURC for its participation in the sea trial. This work was partially financed by FCT, Portugal, under Contract ATOMS, PD-CTM/P/MAR/ 15296/1999 and Project TOMPACO from CNR (Italy). The comments and suggestions of the two anonymous reviewers are greatly appreciated for their contribution to the clarity of the paper.

${ }^{1}$ M. J. Buckingham and S. A. S. Jones, "A new shallow-ocean technique for determining the critical angle of the seabed from the vertical directionality of the ambient noise in the water column," J. Acoust. Soc. Am. 81(4), 938-946 (1987).

${ }^{2}$ M. J. Buckingham and J. R. Potter, "Acoustic daylight imaging: vision in the ocean," GSA Today 4, 97-102 (1994).

N. M. Carbone, G. B. Deane, and M. J. Buckingham, "Estimating the compressional and shear wave speeds of a shallow water seabed from the vertical coherence of ambient noise in the water column," J. Acoust. Soc. Am. 103(2), 801-813 (1998).

${ }^{4} \mathrm{C}$. H. Harrison and A. Baldacci, "Bottom reflection properties by inversion of ambient noise," in Proc. Sixth of European Conf. on Underwater Acoust., ECUA'02, pages 471-476, Gdansk, Poland, June 2002.

${ }^{5} \mathrm{C}$. H. Harrison, "The influence of noise and coherence fluctuations on a new geo-acoustic inversion technique," in Impact of Littoral Environment Variability on Acoustic Predictions and Sonar Performance, edited by N. G. Pace and F. B. Jensen (Kluwer, Dordrecht, 2002), pp. 139-146.

${ }^{6}$ C. H. Harrison and D. G. Simons, "Geoacoustic inversion of ambient noise: A simple method," J. Acoust. Soc. Am. 4(112), 1377-1389 (2002).

M. J. Buckingham, E. M. Giddens, J. B. Pompa, F. Simonet, and T. R. Hahn, "A light aircraft as a source of sound for performing geo-acoustic inversion of the sea bed," in Proc. of Sixth European Conf. on Underwater Acoust., ECUA'02, pages 465-470, Gdansk, Poland, June 2002.

${ }^{8}$ J. A. Cadzow, "Blind deconvolution via cumulant extrema," IEEE Signal Process. Mag. 13, 24-42 (1996).

${ }^{9}$ W. A. Gardner, "A new method of channel identification," IEEE Trans Commun. 39, 813-817 (1991)

${ }^{10} \mathrm{~L}$. Tong, G. $\mathrm{Xu}$, and T. Kailath, "A new approach to blind identification and equalization of multipath channels," in Proc. of the 25th Asilomar Conf. on Signals, Systems and Computers, 1991.
${ }^{11}$ M. K. Broadhead, "Broadband source signature extraction from underwater acoustics data with sparse environmental information," J. Acoust. Soc. Am. 97, 1322-1325 (1995).

${ }^{12}$ M. K. Broadhead and L. A. Pflug, "Performance of some sparseness criterion blind deconvolution methods in the presence of noise," J. Acoust. Soc. Am. 107, 885-893 (2000)

${ }^{13} \mathrm{P}$. Gerstoft, "Inversion of seismoacoustic data using genetic algorithms and a posteriori probability distributions," J. Acoust. Soc. Am. 95(2), 770-782 (1994).

${ }^{14}$ J. -P. Hermand and P. Gerstoft, "Inversion of broad-band multitone acoustic data from the yellow shark summer experiments," IEEE J. Ocean. Eng. 21(4), 324-364 (1996).

${ }^{15}$ J. -P. Hermand, "Broad-band geoacoustic inversion in shallow water from waveguide impulse response measurements on a single hydrophone: Theory and experimental results," IEEE J. Ocean. Eng. 24(1), 41-66 (1999).

${ }^{16}$ M. Siderius, P. L. Nielsen, J. Sellschopp, M. Snellen, and D. Simons, "Experimental study of geo-acoustic inversion uncertainty due to ocean sound-speed fluctuations," J. Acoust. Soc. Am. 110(2), 769-781 (2001).

${ }^{17}$ C. -S. Chiu, J. H. Miller, W. Denner, and J. F. Lynch, "Forward modeling of the barents sea tomography vertical line array data and inversion highlights," in Full Field Inversion Methods in Ocean and Seismo-Acoustics, edited by O. Diachok et al. ( Kluwer Academic, Netherlands, 1995), pp. 237-242.

${ }^{18}$ C. Soares, A. Waldhorst, and S. M. Jesus, "Matched field processing: Environmental focusing and source tracking with application to the north elba data set," in Proc. of the Oceans'99 MTS/IEEE Conference, pages 1598-1602, Seattle, Washington, 13-16 September 1999.

${ }^{19}$ P. Gerstoft and D. Gingras, "Parameter estimation using multi-frequency range-dependent acoustic data in shallow water," J. Acoust. Soc. Am. 99(5), 2839-2850 (1996).

${ }^{20}$ C. Soares, M. Siderius, and S. M. Jesus, "Source localization in a timevarying ocean waveguide," J. Acoust. Soc. Am. 112(5), 1879-1889 (2002).

${ }^{21}$ M. D. Collins and W. A. Kuperman, "Focalization: Environmental focusing and source localization," J. Acoust. Soc. Am. 90(3), 1410-1422 (1991).

${ }^{22}$ C. Soares and S. M. Jesus, "Broadband matched-field processing: Coherent vs incoherent approaches," J. Acoust. Soc. Am. 113(5), 2587-2598 (2003).

${ }^{23}$ S. M. Jesus and C. Soares, "Blind ocean acoustic tomography with source spectrum estimation," in Proc. Int. Conf. on Theoretical and Computational Acoustic, Honolulu, Hawaii, August 2003.

${ }^{24}$ S. M. Jesus, E. Coelho, J. Onofre, P. Picco, C. Soares, and C. Lopes, "The Intifante'00 sea trial: Preliminary source localization and ocean tomography data analysis," in Proc. of the MTS/IEEE Oceans 2001, Honolulu, Hawaii, 5-8 November 2001.

${ }^{25}$ O. C. Rodríguez, S. Jesus, Y. Stephan, X. Démoulin, M. Porter, and E. Coelho, "Internal tide acoustic tomography: Reliability of the normal modes expansion as a possible basis for solving the inverse problem," in Proc. of the 4th. European Conference on Underwater Acoustics, pages 587-592, Rome, Italy, 21-25, September 1998.

${ }^{26} \mathrm{C}$. Soares, "Matched-field processing: Acoustic focalization with data taken in a shallow water area of the Strait of Sicily," MSC Report, SiPLAB-FCT, University of Algarve, Faro, Portugal, March 2001.

${ }^{27}$ C. Soares, M. Siderius, and S. M. Jesus, "High frequency source localization in the Strait of Sicily," in Proc. of the MTS/IEEE Oceans 2001, Honolulu, Hawaii, November 2001.

${ }^{28}$ T. Fassbender, 1995, "Erweiterte genetische algorithmen zur globalen optimierung multi-modaler funktionen, Diplomarbeit, Ruhr-Universität Bochum 1995 .

${ }^{29}$ C. M. Ferla, M. B. Porter, and F. B. Jensen, C-SNAP: Coupled SACLANTCEN normal mode propagation loss model. La Spezia, Italy.

${ }^{30} \mathrm{~S}$. M. Jesus and C. Soares, "Tomografia passiva costiera, inversion results with active data - phase 2," Internal Report Rep. 06/01, SiPLAB/CINTAL, Universidade do Algarve, Faro, Portugal, December 2001.

${ }^{31}$ S. M. Jesus, C. Soares, J. Onofre, E. Coelho, and P. Picco, "Experimental testing of the blind ocean acoustic tomography concept," in Ref. 5, pp. 433-440. 